\title{
Mucosal Reconstruction with Nasal Lateral Wall Flap after Rhinectomy: Two Case Reports
}

\author{
Claudia Esper Zamar, Elián García Pita, Ana Laura Cajelli and Carlos Santiago Ruggeri* \\ Department of Otolaryngology, Hospital Italiano of Buenos Aires, Argentina
}

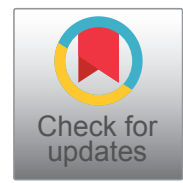

*Corresponding author: Carlos Santiago Ruggeri, MD, Department of Otolaryngology, Hospital Italiano de Buenos Aires - Perón 4190, CP1199, CABA, Argentina

\begin{abstract}
Two patients who had squamous cell carcinomas of the nasal cavity with extension to the nasal septum and previous treatments with surgery and in another with surgery and radiotherapy were treated by rhinectomy.

The reconstruction of the internal mucosal lining was done with mucoperiosteal flaps from the lateral nasal wall with anterior pedicle with the assistance of endoscopes.

There are few descriptions in the literature of the use of this flap to reconstruct the inner lining after rhinectomy.
\end{abstract}

\section{Keywords}

Nasal lateral wall flap, Mucosal reconstruction, Rhinectomy, Endoscopic surgery

\section{Introduction}

The dissection of mucoperiosteal flaps from the nasal cavity has had an important development from the description of the nasoseptal flap by HadadBassagasteguy in 2006 [1].

The main indication was the reconstruction of the skull base after endonasal approaches with endoscopes.

Other mucous flaps were described from the lateral nasal wall, taking advantage of its high vascularity expanding the indications and the sites to be reconstructed $[2,3]$.

The nasal lateral wall flap with anterior pedicle is an option for reconstruction in cases where the nasoseptal flap cannot be used (septum perforations, previous cauterization of the sphenopalatine artery, wide sphenoid antrostomies, etc.), and also for better repair the anterior sectors of the nasal cavity due to its greater range of rotation.

Two patients were treated surgically for squamous cell carcinomas of the nasal cavity, and they were reconstructed with mucoperiosteal flaps from the nasal lateral wall with the anterior pedicle.

There are few descriptions in the literature of the use of this flap to reconstruct the inner lining of the nose after a rhinectomy.

The objective of this presentation of clinical cases is to describe the use of the mucoperiosteal flap of the nasal lateral wall for the reconstruction of the mucosal lining in oncological resections of the nasal pyramid.

\section{Clinical Cases}

\section{Case 1}

Seventy four-year-old man.

He consulted for nasal crusts and mild anterior bilateral epistaxis.

By Computed Tomography (CT), a thickening in the anterior sector of the nasal cavities was diagnosed, with erosion of the premaxilla.

An endonasal biopsy was performed, the histopathological report was squamous cell carcinoma.

It was observed that the tumor involved the anterior mucosa of the nostrils, and the anterior septum in its lower sector.

Under general anesthesia an anterior partial

Citation: Zamar CE, Pita EG, Cajelli AL, Ruggeri CS (2021) Mucosal Reconstruction with Nasal Lateral Wall Flap after Rhinectomy: Two Case Reports. Int J Cancer Clin Res 8:164. doi.org/10.23937/23783419/1410164

Accepted: December 15, 2021: Published: December 17, 2021

Copyright: (C) 2021 Zamar CE, et al. This is an open-access article distributed under the terms of the Creative Commons Attribution License, which permits unrestricted use, distribution, and reproduction in any medium, provided the original author and source are credited. 


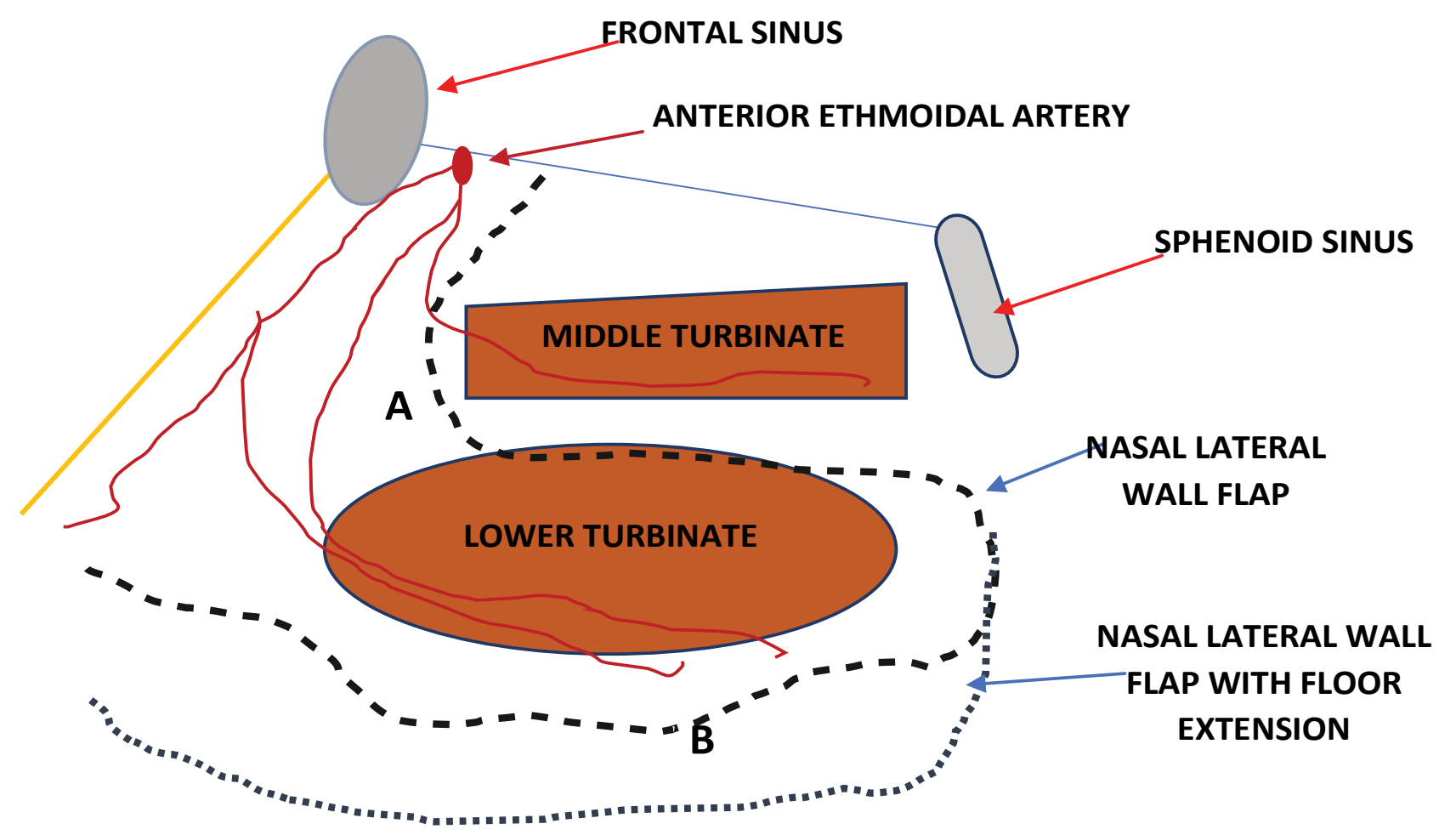

Figure 1: (A) Nasal lateral wall flap with anterior pedicle (anterior ethmoidal artery); (B) Nasal.

maxillectomy and a resection of the anterior mucosa of the floor of both nostrils and the lower and middle part of the nasal septum were performed.

Biopsies of the resection margins were negative for neoplastic cells except for one in the upper sector of the nasal septum.

Due to the need to widen the margin by means of a rhinectomy, it was decided to defer this surgical time to explain the surgery to the patient.

The patient used an obturator to fill the anterior bone defect caused by the partial maxillectomy.

Three weeks later, a partial rhinectomy was performed (resection of the mucosa, anterior septal remnant, columella, part of the alar cartilage: medial, intermediate and part of the lateral cruraand skin of the nasal tip).

Frozen biopsies of the margins were negative.

The defect was reconstructed in three layers: a mucoperiosteal flap was used from lateral wall with anterior pedicle, irrigated by branches of the anterior facial and ethmoidal artery (Figure 1).

The cartilage support was reconstructed with a free ear cartilage graft and the skin of the nasal tip and columella with a middle-frontal flap (Figure 2).

The patient evolved satisfactorily and after a month the pedicle of the middle-frontal flap was sectioned.

He had no recurrences during the one-year followup

\section{Case 2}

Sixty-four year-old male patient.

He had a history of squamous cell carcinoma of the nasal cavity treated 15 months earlier in another center with surgery and postoperative radiotherapy.

The surgery consisted of a anterior partial maxillectomy (premaxilla), resection of the anterior sector of the nasal septum, right middle turbinate and columella.

By CT and magnetic resonance, a tumor was diagnosed in the upper remnant of the nasal septum until the vomer, and involvement of the middle turbinate, anterior and posterior right ethmoids.

She had an episode of posterior epistaxis that did not resolve with an anteroposterior tamponade, for which a digital angiography was indicated. Both sphenopalatine arteries supplying the tumor were embolized.

A biopsy was subsequently done, which was reported as squamous cell carcinoma.

Surgical treatment was indicated.

A total rhinectomy was performed (skin, alar and triangular cartilages, septal remnant, vomer, and nasal mucosa were resected).

The middle turbinate was resected and a wide middle maxillary antrostomy, an anterior and posterior ethmoidectomy, and a right sphenoidotomy were performed using endoscopes. 

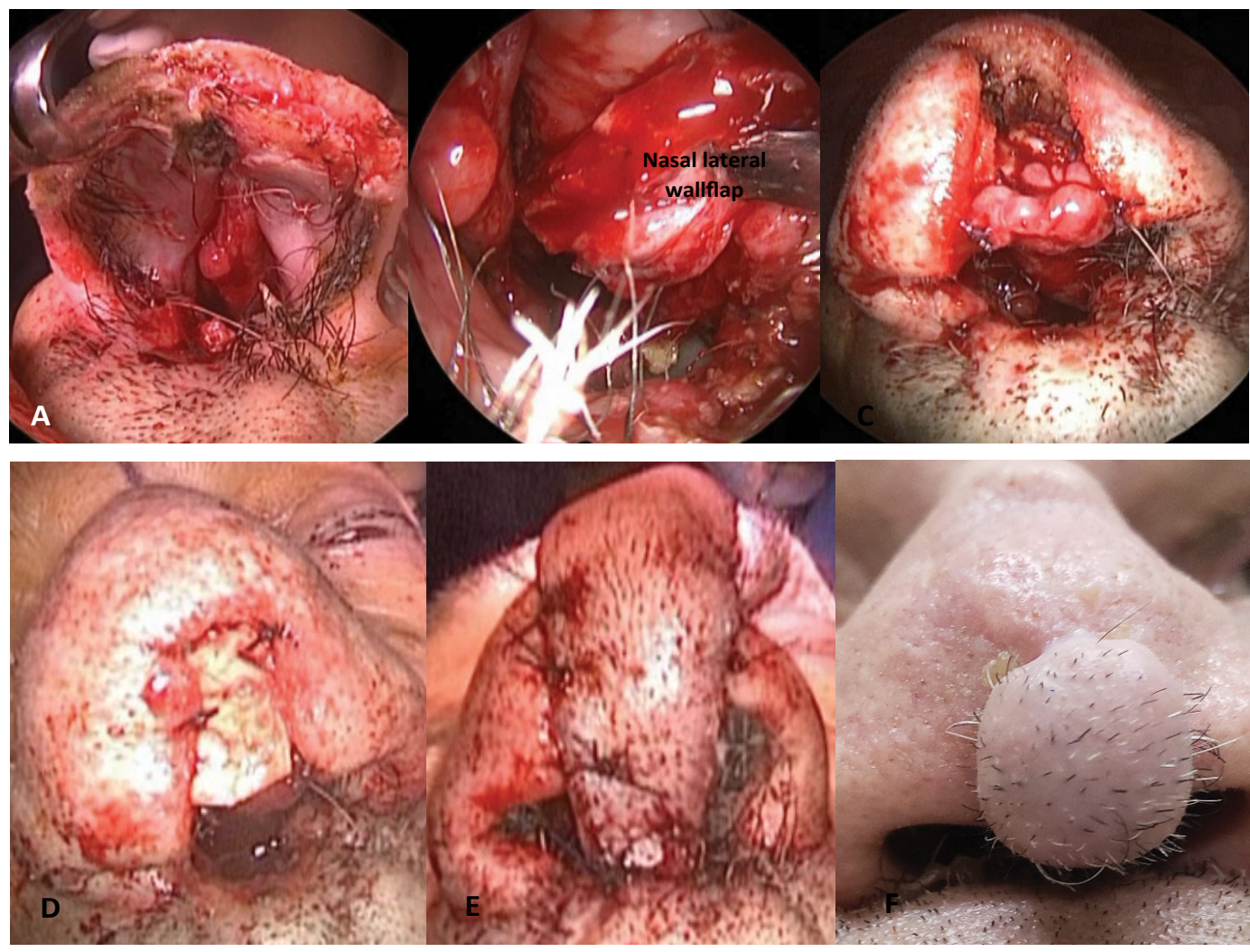

Figure 2: Partial rhinectomy: A- Nasal tip, septum and columella resection, B- Nasal lateral wall flap with anterior pedicle, C- Internal suture of the lateral wall mucoperiosteal flap, D- Ear cartilage suture, E- Nasal tip and columella reconstruction with a middle frontal flap, F- Postoperative at 60 days.

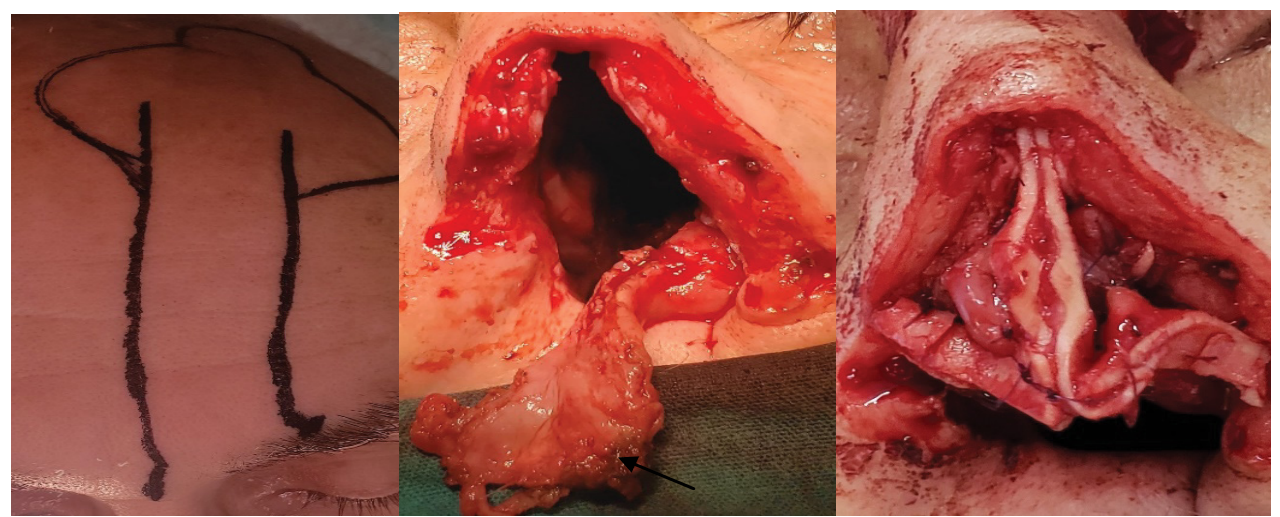

A

B

C

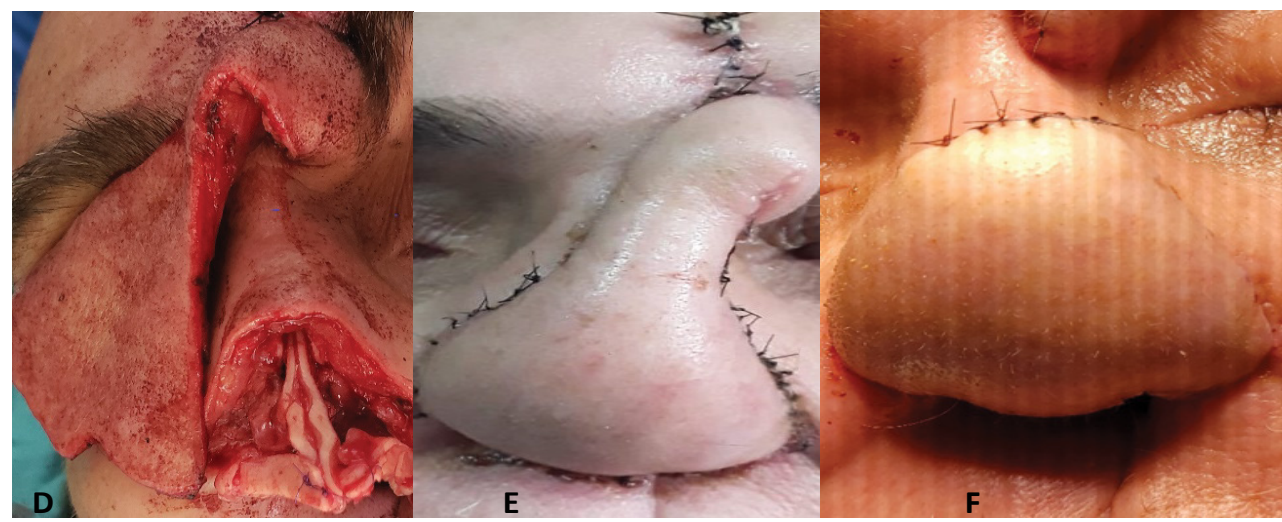

Figure 3: Total rhinectomy: A- Middle-frontal flap design, B- Total rhinectomy and mucoperiosteal flap of nasal lateral wall with extension to the floor with anterior pedicle (arrow), C- Internal suture of the mucoperiosteal flap and cartilaginous reconstruction of the nasal tip with cartilage grafts obtained from the ear, D- Middle frontal flap, E- Posoperative day 7 , FMiddle-frontal flap section of the pedicle at 45 days. 
The frozen biopsies of the resection margins were all negative.

The reconstruction was done in three planes: the mucosa was reconstructed with a lateral wall flap extending to the nasal floor with an anterior pedicle.

With an auricular cartilage graft, a cartilaginous support similar to the alar and triangular cartilages was designed, and the cutaneous lining of the nasal tip and columella was repaired with a medial frontal flap (Figure 3).

He had a good evolution, without complications.

Forty-five days later, the pedicle of the middlefrontal flap was sectioned.

No recurrences were detected during the short follow-up (6 months) that it had.

\section{Discussion}

Basal cell and squamous cell carcinoma originating in the skin of the nose are the most common tumors that can invade the nasal cavity and be treated with surgery [4].

Squamous cell carcinoma originating in the nasal cavity is less common to invade the skin, but the proximity of the compromised mucosa implies a possible invasion of the osseocartilaginous structure of the nasal pyramid and its skin lining.

Treatment with radiotherapy is not the best indication except as palliative therapy in patients who do not accept rhinectomy or in those who do not have an adequate clinical state.

Reconstruction of the nose after partial or total rhinectomy is challenging because the three layers that make up the nose must be reconstructed: skin, osseocartilaginous support, and mucosal lining. The three layers are important and dependent on each other: The internal one depends on the structural support so that collapse does not occur, while the structural support (most often cartilage), depends on the internal and external repair that serve as protection and to vascularize grafts.

There are several reconstruction options for each of these layers.

The middle frontal flap is very useful for repairing large skin defects of the nasal pyramid and columella.

The cartilaginous structure can be reconstructed preferably with auricular or costal cartilage grafts and if there is a need to use bone, this can be obtained from the parietal bone.

The reconstruction of the mucous lining is important to avoid retractions, provide better nasal function and serve as a vascularized bed so that the cartilage grafts survive and do not become necrotic, especially in patients who have had previous treatments with surgery and/or radiotherapy as in the cases described.

To reconstruct the mucosal lining, there are various alternatives that use skin for repair, such as the nasolabial or vestibular flap, the pericranium flap that can be dissected together with the middle-frontal flap or even free flaps such as the radial [5].

Nasal mucoperiosteal flaps such as the nasoseptal or inferior turbinate/lateral wall flaps are ideal because they preserve function and can be dissected unilaterally or bilaterally.

The mucoperiosteum is an ideal tissue, better than the skin to repair mucosal defects, as it is a tissue typical of the nasal cavity and because of its high vascularity.

In the cases described with a single flap the nasal mucosa could be reconstructed and it was possible for the cartilaginous grafts to feed on the vessels of this flap and superficially from the middle frontal flap reducing the possibility of chondronecrosis.

The surgical technique of this flap requires a posterior incision with cautery (curved red tip) at the level of the rhinopharynx behind the tail of the inferior turbinate, then this incision is extended anteriorly below the maxillary ostium and is extended towards the head of the turbinate. Another lower incision that can be extended to the floor of the nasal cavity if more mucosa is required, reaches the anterior sector. Dissection is done in a subperiosteal plane, resecting the turbinate bone, and is facilitated by the use of endoscopes (Figure 1).

The supply of this anterior pedicle flap comes from the branches of the anterior ethmoidal artery and from the lateral nasal artery and angular branches of the facial artery $[3,6]$. For this reason, this flap can still be used in cases of sacrifice of the sphenopalatine artery, as described in the second case.

Langdon, et al. [7] conducted a study in 10 cadavers and reported that with the lateral wall flap they were able to reconstruct defects of approximately $4 \mathrm{~cm}$ long $\times 2.5 \mathrm{~cm}$ wide, and using the flap with extension to the nasal floor they repaired defects of $5 \times 5 \mathrm{~cm}$. They described a clinical case of reconstruction with this flap after a rhinectomy without skin resection.

\section{Conclusions}

Nasal reconstruction after partial or total rhinectomy is complex due to the need to repair different layers of the nasal pyramid.

The lateral wall flap of the nasal cavity with anterior pedicle, with possible extension to the nasal floor depending on the size of the defect to be repaired, is an excellent option for the reconstruction of the internal mucosal lining of the nose. It improves the function and facilitates the nutrition of the cartilage grafts, reducing the possibility of chondronecrosis. 


\section{Conflicts of Interest}

We do not declare to have conflicts of interest.

\section{References}

1. Hadad G, Bassagasteguy L, Carrau RL, Mataza JC, Kassam A, et al. (2006) A novel reconstructive technique after endoscopic expanded endonasal approaches: vascular pedicle nasoseptal flap. Laryngoscope 116: 18821886.

2. Rivera-Serrano CM, Bassagaisteguy LH, Hadad G, Carrau RL, Kelly D, et al. (2011) Posterior pedicle lateral nasal wall flap: New reconstructive technique for large defects of the skull base. Am J Rhinol Allergy 25: 212-216.

3. Hadad G, Rivera-Serrano CM, Bassagaisteguy LH, Carrau RL, Fernandez-Miranda J, et al. (2011) Anterior Pedicle Lateral Nasal Wall Flap: A Novel Technique for the Reconstruction of Anterior Skull Base Defects. Laryngoscope 121: 1606-1610.
4. Girardi FM, Hauth LA, Abentroth AL (2020) Total rhinectomy for nasal carcinomas. Braz J Otorhinolaryngol 86: 763-766.

5. Phillips TJ (2019) Total nasal reconstruction: A review of the past and present, with a peak into the future. Curr Opin Otolaryngol Head Neck Surg 27: 420-425.

6. Mohammad Al-Shouk AA, Tatar I (2021) The blood supply of the inferior nasal concha (turbinate): A cadaveric anatomical study. Anat Sci Int 96: 13-19.

7. Langdon C, Yuen-Ato K, Pereira-Perez C, Arancibia C, Alobid I (2021) Anterior Pedicled Lateral Nasal Wall Flap for Endonasal Lining: A Radiologic and Anatomical Study. Laryngoscope 131: 1462-1467. 